\title{
Direct-to-patient disclosure of results of mismatch repair screening for Lynch syndrome via electronic personal health record: a feasibility study
}

\author{
Michael J. Hall, MD, MS', Meagan M. Herda, BS', Elizabeth A. Handorf, PhD', \\ Christina C. Rybak, CGC', Cindy A. Keleher, PhD ${ }^{1}$, Mark Siemon, BA ${ }^{1}$ and Mary B. Daly, MD, PhD ${ }^{1}$
}

\begin{abstract}
Purpose: The adoption of universal mismatch repair screening of colorectal and endometrial cancers has the potential to improve detection of Lynch syndrome, as well as to improve health outcomes among cancer patients and their family members. Electronic patient health records represent an innovative, resource-efficient route of delivering results directly to patients that could be enhanced by multimedia interventions to improve critical downstream outcomes. The current study examines the feasibility and acceptability of this approach.
\end{abstract}

Methods: Patients hospitalized for resection of colorectal or endometrial cancer were recruited to receive their mismatch repair result via institutional electronic patient health record. Baseline and followup assessments were conducted.

Results: In all, 74\% (49/66) of eligible patients consented, and 81\% $(29 / 36)$ of participants who had a result posted to their electronic patient health record completed follow-up, surpassing feasibility thresholds, with $14 \%$ (5/36) receiving an abnormal result. Ratings of the study approach surpassed the acceptability threshold-97\% had a mean score of $\geq 4$ on a 7 -point scale-and were high, regardless of whether the results were normal or abnormal. Ineligibility was more common among non-white patients $(P=0.009)$ and patients $\geq 65$ of age $(P=0.035)$ due to either low Internet use or access to the Internet.

Conclusion: Electronic patient health record-based result disclosure for mismatch repair screening is feasible to study and is acceptable to patients, but minority and elderly patients may experience greater barriers to participation.

Genet Med advance online publication 1 May 2014

Key Words: electronic patient health record; Lynch syndrome; mismatch repair; universal testing

\section{INTRODUCTION}

Lynch syndrome (LS) is among the most common adult hereditary cancer risk syndromes. ${ }^{1}$ Carrier frequency for mutations in the mismatch repair (MMR) genes associated with LS is $1 / 300$ in the population. ${ }^{2}$ Approximately $3 \%$ of colorectal cancers (CRCs) and $2 \%$ of endometrial cancers (ECs) are related to germ-line mutations in the MMR genes MLH1, MSH2, MSH6, and PMS2, as well as mutations in the EPCAM gene. ${ }^{1-3}$ LS raises lifetime risks of CRC and EC to $60-80 \%$ and $40-60 \%$, respectively, in addition to increasing the lifetime risks of several other cancers. ${ }^{1-3}$ Intensive CRC screening, prophylactic hysterectomy and salpingo-oophorectomy, and high-dose aspirin chemoprevention have been proven to reduce CRC incidence in LS, ${ }^{4-6}$ yet most carriers are unaware that they have LS, leading to many preventable cancers and deaths annually. Improving identification of LS is a public health imperative.?

Research has supported new approaches to improving detection of LS. A 2008 study established the feasibility and cost-effectiveness of universal (i.e., all cases) MMR screening for CRC whereby tumors are routinely tested by immunohistochemistry (IHC) for deficient mismatch repair. ${ }^{8,9}$ A subsequent study reported an uptake rate of $71 \%$ for MMR screening at comprehensive cancer centers but lower uptake in the community.
Substantial variability in procedures to inform patients of their MMR screening results was also observed, but downstream outcomes were not assessed. ${ }^{10}$ Among the concerns raised regarding universal MMR screening is the risk that patients may not pursue genetic counseling and testing when appropriate, diminishing anticipated preventive benefits of screening. A recent retrospective analysis examined three methods to return MMR screening results to patients and found that an unscheduled clinic approach by a genetic counselor (during a medical/ surgical oncology appointment) led to the highest subsequent uptake of germ-line testing. ${ }^{11}$

Effective, resource-efficient methods for disclosing routine genetic screening results to patients are needed. Furthermore, the development of interventions that improve uptake of genetic services among patients and their families following MMR testing is critical to support broad-based implementation of universal MMR screening for LS. Web-based electronic patient health records (ePHRs) are an increasingly common, low-cost adjunct to health care and are in line with patient-centered health care as outlined by the Institute of Medicine. ${ }^{12}$ ePHRs have been positively received by patients and have demonstrated success in facilitating patient-provider communication and in improving access to medical information. ${ }^{13-16}$ Whether it is acceptable 
to allow patients direct access via ePHR to a genetic screening result is unknown. Experience from research and commercial testing suggests that patients have favorable views of online access to genetic results. ${ }^{17,18}$

The current study was developed with the goal of assessing the feasibility and acceptability of a larger study in which routine MMR screening results would be provided via $\mathrm{ePHR}$, accompanied by a multimedia intervention. No previous studies have examined this approach.

\section{MATERIALS AND METHODS Universal MMR tumor screening at the Fox Chase Cancer Center}

IHC staining for MLH1, MSH2, MSH6, and PMS2 expression is conducted on all surgically resected CRC and EC pathological specimens per institutional standard, and all patients receive a handout describing universal MMR screening procedures; the handout includes a toll-free number for the Department of Clinical Genetics. Once testing is complete, a personalized result letter is prepared and provided to the patient's physician, who is then responsible for reviewing it with the patient. All screened patients are tracked prospectively through a clinical database to monitor MMR test completion, provider notification, and follow-up.

\section{MyFoxChase ePHR and study-specific modifications}

All Fox Chase patients are given the option to open a MyFoxChase account (see Supplementary Figure S1a online).
Patients may use the portal to examine their schedule, laboratory results, and to send communications to staff. Patients receive an automated message sent to a linked e-mail account when new results are available. For the study, several ePHR enhancements were made. The automated e-mail message was adapted to indicate that the result of the universal MMR screening test was available. A dedicated electronic results page was also developed to accommodate study needs (see Supplementary Figure S1b,c online); this contained information about LS, as well as contact information for the Department of Clinical Genetics for patients wishing to receive information, schedule an appointment, or speak to a counselor. Adjacent to the results, a "More Information" hot link allowed participants to obtain more-detailed information about LS. Finally, an application to monitor ePHR use was created through which study personnel could track visits to the results page and activation of the "More Information" hot link.

\section{Recruitment}

A schema of study procedures is shown in Figure 1. Patients undergoing resection of a CRC or EC adenocarcinoma were identified using operating room schedules. Patients $\geq 18$ years of age were approached by study personnel 48-72 hours postoperatively. Internet access and use requirements were screened using the following three questions: (i) "Do you have Internet access at home, at work, at another location, or through a personal Smartphone?" (ii) "Do you use the Internet $\geq 1$ time/week?" and (iii) "Do you have an e-mail account that

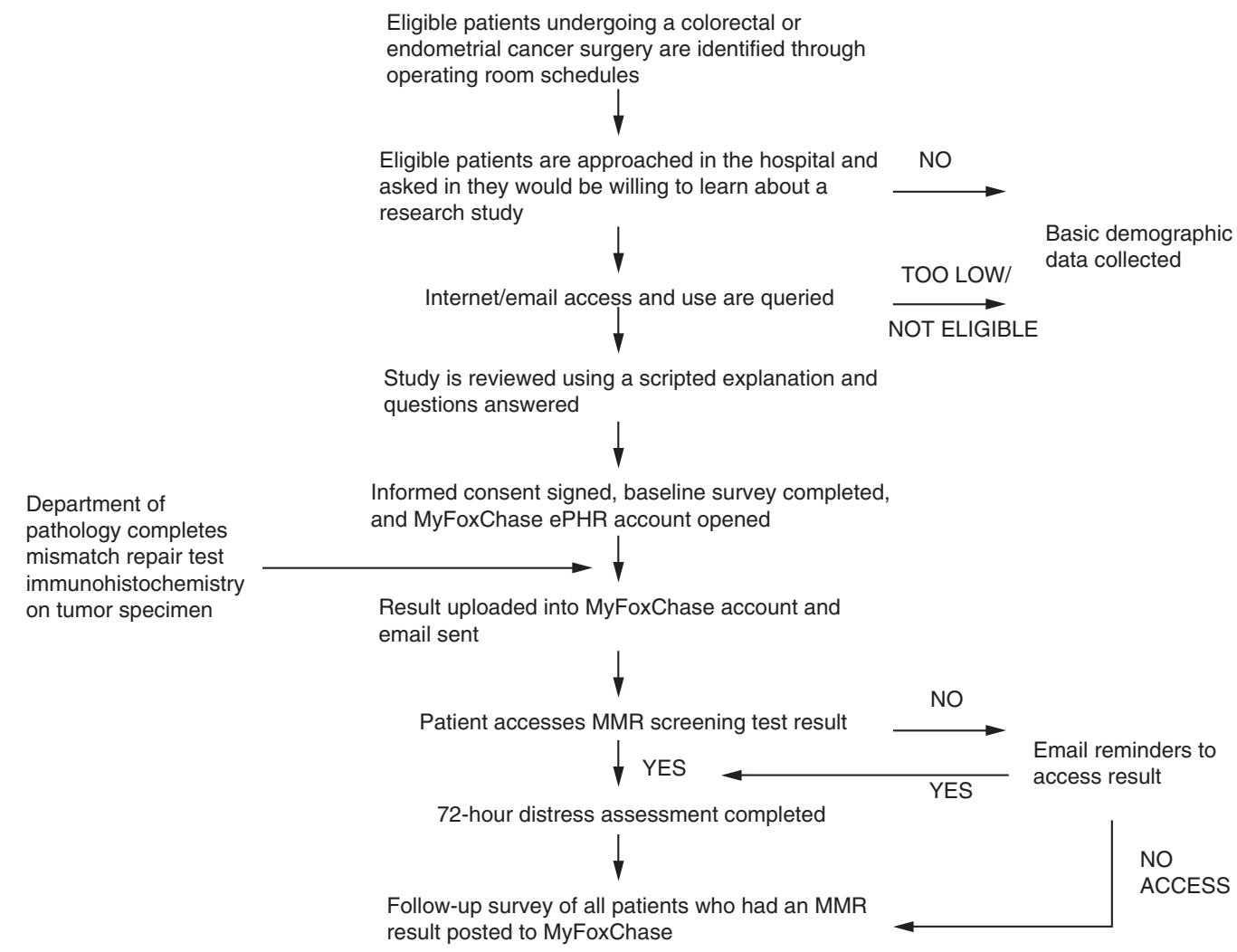

Figure 1 Schema of study procedures. 
you access $\geq 1$ time/week?" The answers to all three questions were required to be positive before proceeding. Patients meeting Internet requirements were briefly introduced to the study using a prepared script and were made aware that they would receive a routine genetic screening result for hereditary cancer risk evaluation via their $\mathrm{ePHR}$ if they agreed to participate.

\section{Study procedures}

Interested patients provided informed consent, completed a baseline survey, and had a MyFoxChase account activated if they did not already have one. Once MMR screening by IHC was completed, the result was posted to the patient's ePHR. Within 36 hours of viewing the result, participants completed a distress measure by telephone. Those who did not view the result within 3 days received e-mail notifications of a pending result. Within 1 month of result posting, participants completed a follow-up survey. Remote monitoring of result viewing and activation of the "More Information" hot link continued for 2 months after posting.

\section{Participant surveys}

All participants completed a baseline survey at enrollment. Demographic characteristics, including age, gender, race, ethnicity, marital status, educational attainment, and household income, were queried. Family history among first- and second-degree relatives was collected, including known cancer syndromes. Distress was assessed at baseline, within 72 hours of result viewing, and at follow-up using the Hospital Anxiety and Depression Scale. ${ }^{19}$ Intention to log in and view the MMR screening result was assessed with one Likert-type item. Location of log-in (e.g., home, work) and discussions with surgical provider regarding the MMR screening result were also examined. Internet privacy preferences were measured using a single item: "How concerned are you about the privacy of personal information on the Internet?" with responses ranging from "not at all" to "very concerned." Perceived risk of hereditary cancer was assessed by two items adapted from previous research, one asking participants to compare the risk of cancer in family members with that of an average person, and the other examining patient perceptions of carrying an altered gene that caused the cancer. ${ }^{20,21}$ Finally, seven Likert-type items (Cronbach's $\alpha=0.74$ ) assessed the acceptability of the study, whereas satisfaction with MMR screening (Cronbach's $\alpha=0.81$ ) was measured using a 10 -item scale, both adapted from our previous research. ${ }^{22-24}$

\section{Statistical methods}

We summarized characteristics of all patients, consented patients, and analyzable patients using percentages and frequency tables. We looked for differences between patient groups (those who were not eligible or did not consent versus consented patients; consented but not analyzable versus analyzable patients) using Fisher's exact test. Survey data were summarized using frequency tables, proportions, means, and SDs. Feasibility and acceptability thresholds were set by the investigators and were informed by previous behavioral intervention research in cancer patients and low-risk primary-care patients. ${ }^{22-25}$ Feasibility was determined by the consent rate among eligible patients, with a rate of $\geq 50 \%$ deemed feasible to complete accrual of a two- to three-center trial within 3 years, and by the rate of completion of all study components, with a rate of $\geq 75 \%$ deemed acceptable to support the successful analysis of outcomes in a future study. Acceptability of the study approach and procedures was determined by a subjective seven-item acceptability measure (see above) adapted from our previous research. ${ }^{22-24} \mathrm{~A}$ mean acceptability rating of 4 or greater in $\geq 75 \%$ participants was deemed a minimum necessary threshold.

\section{Recruitment}

\section{RESULTS}

A recruitment flow diagram is shown in Figure 2, and characteristics of the population available for recruitment (total pool), those who signed consent (consented), and those who had a result posted to their ePHR (analyzable) are shown in Table 1. Over a period of 6 months, 131 patients with CRC or EC were identified, and $91 \%$ were successfully approached during their hospitalization. Overall, 65 (54\%) patients were ineligible at recruitment, more than half $(34 / 65,54 \%)$ because of either lack of Internet access or use of the Internet/e-mail $<1$ time/ week. Non-white patients were more likely than white patients to be ineligible $(P=0.009)$, and those $\geq 65$ years of age were more likely to be ineligible $(P=0.035)$. Other reasons for ineligibility included no diagnosis of cancer $(n=26)$ and previous MMR screening for LS $(n=5)$. Of the remaining 66 patients, $26 \%$ refused participation in the study, many indicating low interest. Patients either refused actively $(n=10)$ at recruitment or did not contact personnel after taking an informational flyer $(n=7)$. Patients $\geq 65$ years of age were more likely to refuse participation than were those aged $<65$ years of age $(P=0.003)$.

\section{Consented population}

The mean age of consented participants was 59 years, and $76 \%$ of these were female. Nearly all were white (Asian ancestry, $n=1$ ), and $6 \%$ reported Hispanic ethnicity. Educational attainment and income levels were diverse-29\% had a high school diploma or less, whereas $53 \%$ were college graduates. Reported household income was $\$ 50,000-100,000$, but this was $<\$ 50,000$ for $22 \%$ of the participants. More than two-thirds (68\%) had more than one first-degree relative with cancer, and one participant reported a family history of LS. Perceived risk levels were low to moderate, with nearly $90 \%$ estimating CRC risk in relatives as "the same" or "a little higher" compared with others, and more than half (61\%) estimating the chances that an altered gene had caused their cancer as "not at all" or "a little." Finally, 69\% were "moderately concerned" or "very concerned" about Internet privacy, but there were no differences in degree of concern by demographic factors.

\section{Feasibility and acceptability}

The feasibility thresholds for consent rate and completion rate were met. Overall, 49/66 (74\%) eligible patients consented to 
All patients with a scheduled surgical resection of a colorectal and endometrial tumor upon which immunohistochemistry (IHC) screening for defective mismatch repair is routinely performed at FCCC $(n=131)$

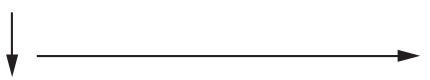

Successfully approached for recruitment $(n=121)$

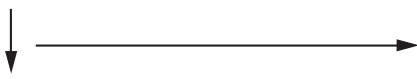

Eligible for recruitment to the study $(n=66)$

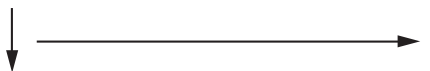

Consented to the study and completed the baseline survey $(n=49)$

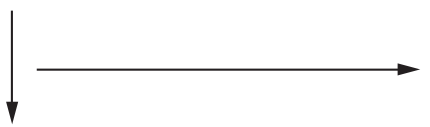

Analyzable for study outcomes $(n=36)$

Viewed online result and completed follow-up survey $(n=29)$

Did not view result but completed follow-up survey $(n=2)$

Viewed online result but did not complete follow-up survey $(n=3)$

Did not view result or complete follow-up survey $(n=2)$

Figure 2 Flow diagram of recruitment. FCCC, Fox Chase Cancer Center.

the study, surpassing the first feasibility benchmark of a $\geq 50 \%$ consent rate. From among these 49 participants, 13 (27\%) were later removed from the study-10 were removed due to insufficient tumor samples to complete MMR screening, and the first 3 participants enrolled were removed due to technical failure of the ePHR results display-leaving 36/49 (73\%) participants who had their MMR screening result posted to their ePHR. From among the participants in this group, 32/36 (89\%) viewed the ePHR, and $31 / 36(86 \%)$ completed the follow-up surveys (of note, 29/36 (81\%) participants both viewed their result and completed the follow-up surveys). At $81 \%$, this completion rate exceeded the second feasibility threshold of $\geq 75 \%$. Notably, $5 / 36$ (14\%) participants had an abnormal result.

Participant-rated acceptability of the study was high $-97 \%$ had a mean score of $\geq 4$ on a 7 -point scale (Table 2 ). There was no difference in acceptability by MMR screening result (normal result: mean $=6.4(\mathrm{SD}=0.6)$; abnormal result: mean $=6.2$ $(\mathrm{SD}=1.4), P=0.7)$. Acceptability was higher among married participants $(P=0.01)$. Satisfaction with MMR screening was also high for those receiving either a normal (mean $=3.08$, $\mathrm{SD}=0.51$ ) or an abnormal (mean $=3.12, \mathrm{SD}=0.80$ ) result. Acceptability $(P=0.08)$ and satisfaction $(P=0.006)$ were borderline and were significantly higher among participants who, at baseline, reported higher perceived risk of carrying an altered gene that caused their cancer.

\section{Analyzable population outcomes}

All participants viewed their MMR screening result by ePHR at home. Married participants $(P=0.05)$ and those with abnormal $(P=0.06)$ or borderline results were more
Unable to approach for recruitment prior to discharge from hospital $(n=10)$

Ineligible $(n=65)$ due to no Internet access, low use, or lack of an email account $(n=34)$, surgery not performed $(n=16)$, or already tested for Lynch syndrome $(n=5)$

Declined to participate $(n=17)$

Completed baseline but not included in outcome analyses due to no tumor available in surgical

specimen to perform IHC testing $(n=10)$ or technical failure of the personal health record (PHR) Web interface to release the test result $(n=3)$ likely to view their result multiple times (range: two to six times). The majority viewed their result $<24$ hours after posting $(22 / 32,69 \%)$, whereas others took up to 18 days. The "More Information" hot link was activated by 25/32 (78\%) participants, including 4 of the 5 participants with abnormal results. Among those with a normal result, married participants were significantly more likely to activate the "More Information" hot link $(P=0.004)$. The only participant with an abnormal result who did not activate the "More Information" hot link had a family history of LS. The results for ePHR use are shown in Table 3.

Overall, 63.6 and $60 \%$ of participants receiving normal and abnormal results, respectively, reported a discussion of their result with their doctor. Among those with an abnormal result, three of the five participants contacted the Department of Clinical Genetics, and two of these three scheduled an appointment, were counseled, and were tested; the remaining two participants (of the five with abnormal results) received counseling concurrent with a medical oncology appointment, and one of these pursued further testing, whereas the other, a 23-year-old patient with CRC and a family history of LS, has yet to pursue testing. None who received a normal MMR screening result have pursued genetic evaluation at the Fox Chase Cancer Center.

\section{Anxiety monitoring}

No overall differences were seen between anxiety levels at baseline and at 72 hours or for those receiving a normal versus those receiving an abnormal result. Anxiety levels at follow-up were significantly lower than those at baseline $(P=0.003)$. 
Table 1 Characteristics of postoperative endometrial and colorectal cancer patients undergoing MMR screening and comparison of nonconsented versus consented populations

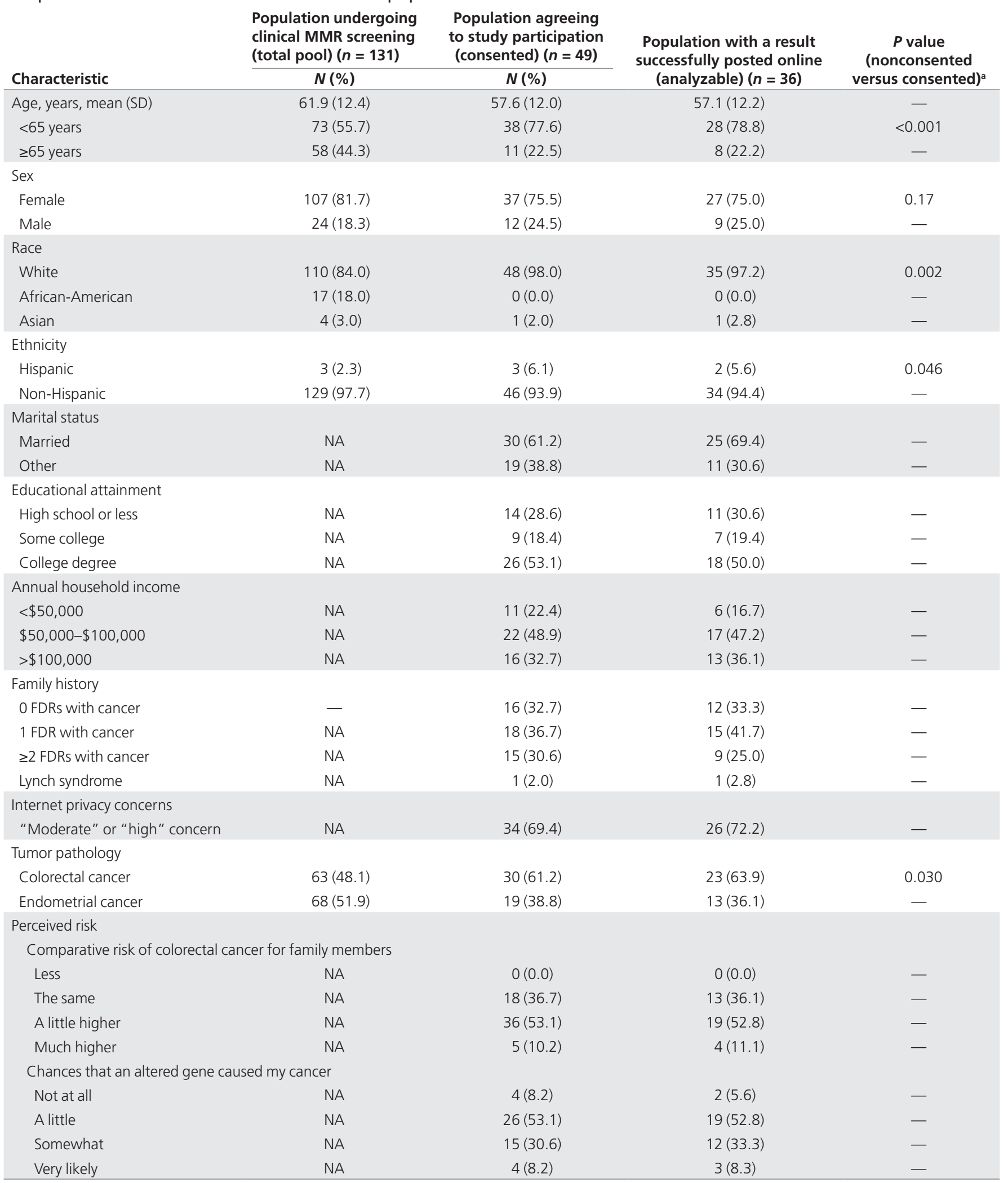

FDR, first-degree relative; MMR, mismatch repair; NA, not applicable.

aComparison of those in the total pool who did not consent $(n=82)$ versus those who did consent $(n=49)$. There were no significant $(P<0.05)$ differences between the analyzable cohort $(n=36)$ and those who consented but were not analyzable $(n=13)$. 


\section{DISCUSSION}

Underrecognition of LS leads to thousands of preventable cancer diagnoses annually., ${ }^{2,3}$ Universal tumor-based MMR screening of CRCs and ECs has shifted the paradigm for identifying LS among cancer patients, but it remains uncertain how to efficiently inform patients of the result from this routine screening test for hereditary cancer risk. To maximize dissemination potential, novel methods to disclose genetic results to patients must consider resource/time constraints, patient preference, and the effectiveness of the method in promoting positive downstream behavioral outcomes. ${ }^{2,3,8-10}$

Table 2 ePHR use among participants who had an MMR screening result successfully posted to their electronic health record (analyzable population)

\begin{tabular}{lcc}
$\begin{array}{l}\text { Study component } \\
\text { Result posted to } \\
\text { ePHR ( } n=36)\end{array}$ & $\begin{array}{c}\text { All participants } \\
\text { who had a result } \\
\text { posted, } \boldsymbol{n} \text { total (\%) }\end{array}$ & $\begin{array}{c}\text { Participants who } \\
\text { received an abnormal } \\
\text { result, } \boldsymbol{n} \text { /total (\%) }\end{array}$ \\
$\begin{array}{l}\text { online } \\
\text { Result viewed online } \\
(n=32)\end{array}$ & $36 / 36(100)$ & $5 / 5(100)$ \\
$\begin{array}{l}\text { Viewed one to two } \\
\text { times }\end{array}$ & $18 / 32(56.3)$ & $5 / 5(100)$ \\
$\begin{array}{l}\text { Viewed three or } \\
\text { more times }\end{array}$ & $13 / 32(40.6)$ & $n=5$ \\
$\begin{array}{l}\text { Activation of "More } \\
\text { Information" tab }\end{array}$ & $25 / 32(78.1)$ & $1 / 5(20.0)$ \\
$\begin{array}{l}\text { Did not activate } \\
\text { Activated one time }\end{array}$ & $6 / 32(18.8)$ & $4 / 5(80.0)$ \\
$\begin{array}{l}\text { Activated two to } \\
\text { four times }\end{array}$ & $14 / 32(43.8)$ & $1 / 5(20.0)$ \\
\hline $\begin{array}{l}\text { Discussion of result } \\
\text { with provider }\end{array}$ & $20 / 32(62.5)$ & $0 / 5(0.0)$ \\
\hline ePHR, electronic patient health record; MMR, mismatch repair.
\end{tabular}

The current study examines the provision of patient access to universal MMR screening results via ePHR and was primarily designed to evaluate the feasibility and acceptability of a future clinical trial to evaluate the impact of this novel approachwhen additionally enhanced by a multimedia educational intervention-on psychological and behavioral outcomes. Secondary goals included assessment of how participants used the ePHR result interface and of anxiety related to receipt of the result, as well as exploratory assessment of psychological factors that may affect desirable downstream behaviors (e.g., uptake of genetic counseling and testing). We demonstrate that a future study is feasible, with robust consent $(74 \%)$ and study component completion (81\%) rates surpassing target thresholds. We nonetheless acknowledge that universal testing protocols at some centers may include a more comprehensive testing approach than the one examined in our pilot study. For example, many centers additionally conduct testing for the BRAF V600E mutation in CRC specimens that demonstrate loss of the MLH1 and PMS2 protein staining by IHC because presence of this mutation is strongly and negatively associated with LS. To improve the generalizability of our pilot approach to the community-based cancer setting wherein onsite molecular pathology facilities may not be available, we chose to focus on the results of the IHC test. However, we believe centers with more comprehensive universal MMR testing protocols could develop ePHR-based notification modules similar to the one examined here, that compile and report results of all elements of testing performed in a summary format. Although the reporting of these additional tests may increase the complexity of the final report, patients could be given access to both (i) immediate online support through "More Information" hot links, as was done in the current study, and (ii) clinical support as needed, through their providers.

Our findings suggest potential age- and race-related recruitment barriers to studies involving Internet interventions. Nonwhite, predominantly African-American patients $(P=0.009)$ and patients $\geq 65$ years of age $(P=0.035)$ were more likely to

Table 3 Acceptability and satisfaction among participants completing the follow-up survey, stratified by MMR screening result $(n=31)$

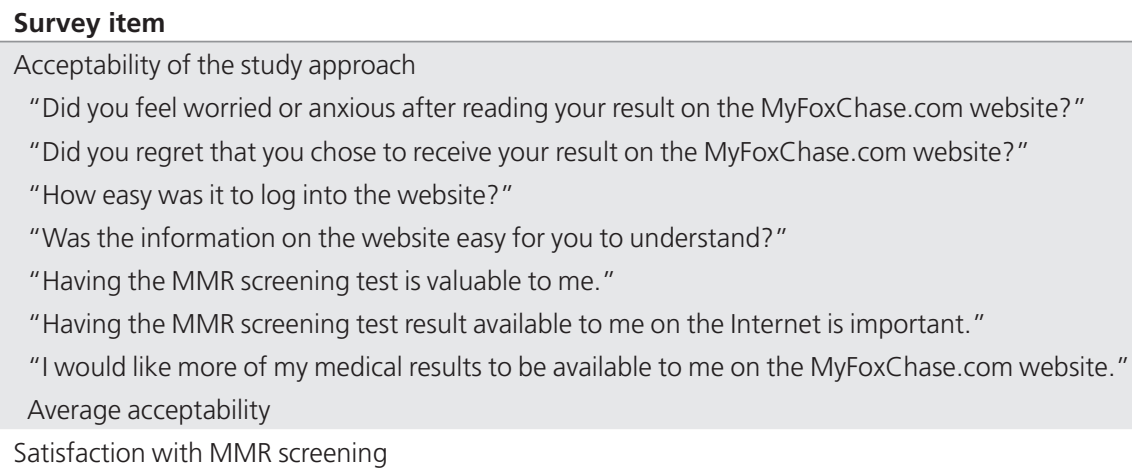

\begin{tabular}{|c|c|c|}
\hline $\begin{array}{l}\text { Normal result } \\
(n=26, \text { range: } \\
1-7) \text {, mean (SD) }\end{array}$ & $\begin{array}{c}\text { Abnormal result } \\
(n=5, \text { range: } \\
1-7), \text { mean (SD) }\end{array}$ & $\begin{array}{c}P \\
\text { value }\end{array}$ \\
\hline $6.7(0.6)$ & $6.2(1.8)$ & 0.24 \\
\hline $6.8(0.5)$ & $6.2(1.8)$ & 0.14 \\
\hline $6.4(1.3)$ & $6.4(1.3)$ & 0.99 \\
\hline $5.9(1.5)$ & $6.6(0.9)$ & 0.32 \\
\hline $6.6(0.7)$ & $5.8(1.6)$ & 0.07 \\
\hline $6.2(1.0)$ & $5.8(1.6)$ & 0.46 \\
\hline $6.2(1.6)$ & $6.6(0.9)$ & 0.59 \\
\hline 6.4 & 6.2 & 0.7 \\
\hline
\end{tabular}

$3.08(0.05)$ 
be ineligible for this study due to inadequate Internet access or use. Recent figures from the Pew Research Council show that Internet use is steadily growing among older Americans and minority households but remains lower than that recorded for younger adults and whites. ${ }^{26}$ Age-related differences in Internet access/use among CRC and EC patients are likely to diminish in the coming years as Web-savvy young adults enter middle adulthood. Moreover, high levels of Smartphone use among young, non-white adults further support the transience of age- and race-related barriers. ${ }^{27}$

Eligible patients $\geq 65$ years of age were also more likely to refuse study participation, and many indicated low interest as their reasoning. Here, low interest is challenging to elucidate because it may indicate low interest either in any research or specifically in Internet-based or genetics research. Older patients have been shown to be less likely to participate in therapeutic clinical trials, but this often reflects stringent eligibility criteria and provider biases. ${ }^{28,29}$ Interestingly, Internet privacy preferences and acceptability did not vary by age; however, it remains possible that older patients refused participation out of concern for privacy or due to age-related barriers to ePHRs. ${ }^{30,31}$ Poor understanding of and negative attitudes toward genetics may also have diminished interest among older patients. ${ }^{32,33}$

A growing body of research has begun to examine disclosure of genetic test results via the Internet. ${ }^{34-37}$ Our study is innovative in that it examines, in a manner largely impossible to replicate in a high-risk clinic, disclosure of a routine genetic screening result for hereditary cancer predisposition following a cancer surgery in an unselected, average-risk population with low awareness of MMR testing and LS. As seen in a growing number of cancer centers, universal MMR screening at the Fox Chase Cancer Center is performed automatically and does not require informed consent, ${ }^{10,38}$ and thus the total patient pool for recruitment was unbiased by perceptions of risk or attitudes toward genetics. Furthermore, in an effort to reduce selection bias rooted in perceived risk, our recruiters emphasized the goal of examining a novel route (ePHR) to deliver a standardof-care genetic screening result (i.e., a nondiagnostic result).

Patient perceptions of ePHRs are positive, and studies have shown robust use, despite concerns about Internet privacy. ${ }^{39,40}$ It remains unclear nonetheless whether any genetic results are appropriate for ePHRs, and the research described here breaks new ground in examining a routine genetic screening test for hereditary cancer. Further supporting feasibility, several findings are probably reflective of shifting public attitudes toward genetics and privacy: $89 \%$ of participants viewed their MMR screening result via ePHR, and acceptability ratings were high despite low to moderate perceived genetic risk of cancer and regardless of whether a normal or abnormal result was received. Expanding use of the Internet in the population and favorable public attitudes toward Internet-based medicine and genetics also support the notion that ePHRs could be dually harnessed to deliver MMR screening results as well as to facilitate subsequent steps in a patient's evaluation. For example, direct-toprovider messaging and/or scheduling functions embedded in the ePHR could permit patients to request or initiate a consultation with a genetic counselor. In the future, greater integration of ePHRs into clinical practice may also increase efficiency in the transmission of genetic risk information to patients. Patients receiving a normal screening result will benefit from the information that appropriate screening for LS was performed on their tumor but will be less likely to require a lengthy discussion of this result with their provider because many will have reviewed information made available to them through the ePHR before the clinic visit. Those patients receiving an abnormal screening result will, alternatively, have the opportunity to review the materials made available through resources such as "More Information" links, supplementing their preparation for the discussion with their provider and allowing for a more focused discussion of their result and suggested next steps.

The study has several important limitations. As discussed earlier, center-specific protocols for universal MMR screening may be different from the one examined here-for example, additional testing for BRAF V600E and MLH1 methylation may be reflexively performed after IHC-and thus our approach would need to be carefully adapted to the standard procedures at a given institution. Second, our rate of ineligibility was higher than anticipated, resulting in a sample size inadequate to test for race- or ethnicity-based or other subgroup differences in acceptability and outcomes, although these were not primary goals of this research. Third, because of the novelty of the approach and the genetic nature of the information, we felt in-person recruitment and informed consent were critical while recognizing that this approach was not fully representative of a real-world setting. Indeed, outside of a study, one potential option would be that patients activating an ePHR account would have the ability to establish personal preferences related to access to particular types of informationthus, they could elect to either view or not view results that may be considered sensitive, such as universal MMR screening. The limited information provided about the study during recruitment and informed consent may also have biased the consented population toward patients with greater concerns about hereditary cancer risk. Finally, the Fox Chase Cancer Center is a tertiary care cancer center, and thus the surgical population is not generalizable to a community-based setting; however, the education and income levels among study participants demonstrate notable heterogeneity.

In conclusion, our study supports the notion that routine genetic screening results may be made available to cancer patients via Web-based patient-oriented ePHRs. Patients are interested in receiving genetic screening results online and view this approach as valuable whether they receive a positive or negative result. As the number of genetic markers and tests in medicine grows, further research to study the Internet as a means to provide patients direct access to their genetic results is needed. On the basis of our findings, a future randomized clinical trial will examine whether a Web-based educational intervention, when coupled with the MMR screening result, may improve critical downstream outcomes of MMR screening, 
including uptake of genetic testing among patients and family members at risk for LS.

\section{SUPPLEMENTARY MATERIAL}

Supplementary material is linked to the online version of the paper at http://www.nature.com/gim

\section{ACKNOWLEDGMENTS}

This research was supported in part by funding received through the Fox Chase Cancer Center core grant from the National Cancer Institute (P30 006927-45). M.J.H. is the recipient of a Mentored Research Scholar Grant from the American Cancer Society (MRSG-07-232-01-CPHPS).

\section{DISCLOSURE}

The authors declare no conflict of interest.

\section{REFERENCES}

1. Jasperson K, Tuohy T, Neklason D, Burt R. Hereditary and familial colon cancer. Gastroenterology 2010;138:2044-2058.

2. Boland C, Shike M. Report from the Jerusalem workshop on Lynch syndromehereditary nonpolyposis colorectal cancer. Gastroenterology 2010;138:2197. e2191-2197.

3. Palomaki GE, McClain MR, Melillo S, Hampel HL, Thibodeau SN. EGAPP supplementary evidence review: DNA testing strategies aimed at reducing morbidity and mortality from Lynch syndrome. Genet Med 2009;11:42-65.

4. Järvinen $\mathrm{HJ}$, Aarnio $\mathrm{M}$, Mustonen $\mathrm{H}$, et al. Controlled 15 -year trial on screening for colorectal cancer in families with hereditary nonpolyposis colorectal cancer. Gastroenterology 2000;118:829-834.

5. Lynch HT, Casey MJ. Prophylactic surgery prevents endometrial and ovarian cancer in Lynch syndrome. Nat Clin Pract Oncol 2007;4:672-673.

6. Burn J. Long-term effect of aspirin on cancer risk in carriers of hereditary colorectal cancer: an analysis from the CAPP2 randomised controlled trial. The Lancet 2011;378:2081-2087.

7. HealthyPeople.gov. http://www.healthypeople.gov/2020/default.aspx.

8. Hampel H, Frankel WL, Martin E, et al. Feasibility of screening for Lynch syndrome among patients with colorectal cancer. J Clin Oncol 2008;26: 5783-5788.

9. Mvundura M, Grosse SD, Hampel H, Palomaki GE. The cost-effectiveness of genetic testing strategies for Lynch syndrome among newly diagnosed patients with colorectal cancer. Genet Med 2010;12:93-104.

10. Beamer LC, Grant ML, Espenschied CR, et al. Reflex immunohistochemistry and microsatellite instability testing of colorectal tumors for Lynch syndrome among US cancer programs and follow-up of abnormal results. J Clin Oncol 2012;30:1058-1063.

11. Heald B, Plesec T, Liu X, et al. Implementation of universal microsatellite instability and immunohistochemistry screening for diagnosing lynch syndrome in a large academic medical center. J Clin Oncol 2013;31:1336-1340.

12. Institute of Medicine. Crossing the Quality Chasm: A New Health System for the 21st Century. http://www.iom.edu/ /media/Files/Report\%20Files/2001/ Crossing-the-Quality-Chasm/Quality \%20Chasm \%202001\%20\%20 report\%20brief.pdf.

13. Frost JH, Massagli MP. Social uses of personal health information within PatientLikeMe, an online patient community: what can happen when patients have access to one another's data. J Med Internt Res 2008;10:e15.

14. Lobach DF, Willis JM, Macri JM, Simo J, Anstrom KJ. Perceptions of Medicaid beneficiaries regarding the usefulness of accessing personal health information and services through a patient internet portal. AMIA Annu Symp Proc 2006;509-513.

15. Simon SR, Evans JS, Benjamin A, Delano D, Bates DW. Patients' attitudes toward electronic health information exchange: qualitative study. J Med Internet Res 2009;11:e30.

16. Beckjord $\mathrm{E}$. Use of the Internet to communicate with health care providers in the United States: estimates from the 2003 and 2005 Health Information National Trends Survey (HINTS). J Med Internet Res 2007;9:e20.
17. Leighton JW, Valverde K, Bernhardt BA. The general public's understanding and perception of direct-to-consumer genetic test results. Public Health Genomics 2012;15:11-21.

18. Gollust SE, Gordon ES, Zayac C, et al. Motivations and perceptions of early adopters of personalized genomics: perspectives from research participants. Public Health Genomics 2012;15:22-30.

19. Zigmond AS, Snaith RP. The hospital anxiety and depression scale. Acta Psychiatr Scand 1983;67:361-370.

20. Lerman C, Masny A, Balshem A. Attitudes about genetic testing for breastovarian cancer susceptibility. J Clin Oncol 1994;12:843-850.

21. Lerman C, Biesecker B, Benkendorf JL, et al. Controlled trial of pretest education approaches to enhance informed decision-making for BRCA1 gene testing. J Natl Cancer Inst 1997;89:148-157.

22. Hall MJ, Manne SL, Winkel G, Chung DS, Weinberg DS, Meropol NJ. Effects of a decision support intervention on decisional conflict associated with microsatellite instability testing. Cancer Epidemiol Biomarkers Prev 2011;20:249-254.

23. Manne SL, Meropol NJ, Weinberg DS, et al. Facilitating informed decisions regarding microsatellite instability testing among high-risk individuals diagnosed with colorectal cancer. J Clin Oncol 2010;28:1366-1372.

24. Manne SL, Chung DC, Weinberg DS, et al. Knowledge and attitudes about microsatellite instability testing among high-risk individuals diagnosed with colorectal cancer. Cancer Epidemiol Biomarkers Prev 2007;16:2110-2117.

25. Myers RE, Weinberg DS, Manne SL, et al. Genetic and environmental risk assessment for colorectal cancer risk in primary care practice settings: a pilot study. Genet Med 2007;9:378-384.

26. Wyatt E. Most of U.S. Is Wired, but Millions Aren't Plugged In. The New York Times. http://www.nytimes.com/2013/08/19/technology/a-push-to-connectmillions-who-live-offline-to-the-internet.html?_r=0.

27. Zickuhr K, Madden M. Older adults and internet use.http://www. pewinternet. org/Reports/2012/Older-adults-and-internet-use.aspx.

28. Unger JM, Hershman DL, Albain KS, et al. Patient income level and cancer clinical trial participation. J Clin Oncol 2013;31:536-542.

29. Kemeny $M M$, Peterson $B L$, Kornblith $A B$, et al. Barriers to clinical trial participation by older women with breast cancer. J Clin Oncol 2003;21: 2268-2275.

30. MarketingCharts staff. 9 in 10 Americans Concerned About Online Privacy. http://www.marketingcharts.com/wp/direct/9-in-10-americans-concernedabout-online-privacy-21156/.

31. Wen K, Kreps G, Zhu F, Miller S. Consumers' perceptions about and use of the internet for personal health records and health information exchange: analysis of the 2007 Health Information National Trends Survey. J Med Internet Res 2010;12:e73.

32. Kinney AY, Choi YA, DeVellis B, Millikan R, Kobetz E, Sandler RS. Attitudes toward genetic testing in patients with colorectal cancer. Cancer Pract 2000;8:178-186.

33. Andrykowski MA, Lightner R, Studts JL, Munn RK. Hereditary cancer risk notification and testing: how interested is the general population? J Clin Oncol 1997;15:2139-2148.

34. O'Neill SC, White DB, Sanderson SC, et al. The feasibility of online genetic testing for lung cancer susceptibility: uptake of a web-based protocol and decision outcomes. Genet Med 2008;10:121-130.

35. Sanderson SC, O'Neill SC, White DB, et al. Responses to online GSTM1 genetic test results among smokers related to patients with lung cancer: a pilot study. Cancer Epidemiol Biomarkers Prev 2009;18:1953-1961.

36. McBride CM, Alford SH, Reid RJ, Larson EB, Baxevanis AD, Brody LC. Characteristics of users of online personalized genomic risk assessments: implications for physician-patient interactions. Genet Med 2009;11: 582-587.

37. Stack CB, Gharani N, Gordon ES, Schmidlen T, Christman MF, Keller MA. Genetic risk estimation in the Coriell Personalized Medicine Collaborative. Genet Med 2011;13:131-139.

38. Bellcross CA, Bedrosian SR, Daniels E, et al. Implementing screening for Lynch syndrome among patients with newly diagnosed colorectal cancer: summary of a public health/clinical collaborative meeting. Genet Med 2012;14:152-162.

39. Zulman DM, Nazi KM, Turvey CL, Wagner TH, Woods SS, An LC. Patient interest in sharing personal health record information: a web-based survey. Ann Intern Med 2011;155:805-810.

40. Hassol A, Walker JM, Kidder D, et al. Patient experiences and attitudes about access to a patient electronic health care record and linked web messaging. J Am Med Inform Assoc 2004;11:505-513. 